\title{
High-Dose Intravenous Immunoglobulin Exerts Its Beneficial Effect in Patients with Dermatomyositis by Blocking Endomysial Deposition of Activated Complement Fragments
}

\author{
Milan Basta* and Marinos C. Dalakas ${ }^{\ddagger}$ \\ * Laboratory of Clinical Investigation, National Institute of Allergy and Infectious Diseases; and the ${ }^{\ddagger}$ Medical Neurology Branch, \\ National Institute of Neurological Disorders and Stroke, National Institutes of Health, Bethesda, Maryland 20892
}

\begin{abstract}
In patients with dermatomyositis (DM) the earliest lesion is microvasculopathy mediated by deposition of C5b-C9 membranolytic attack complex (MAC) on intramuscular capillaries. This leads sequentially to muscle ischemia, necrosis of muscle fibers, and muscle weakness. High-dose intravenous immunoglobulin (IVIG), which can modulate complement-dependent tissue damage in animal models, has been shown to be effective in the treatment of patients with DM. We used an in vitro C3 uptake assay to examine 55 coded sera from 13 patients with $D M$ and 5 patients with other non-complement-mediated neuromuscular diseases, before and after treatment with IVIG or placebo. Patients with active DM had a significantly higher baseline C3 uptake compared with the others (geometric mean 12,190 vs $3,090 \mathrm{cpm})$. Post-IVIG but not post-placebo sera inhibited the $\mathrm{C} 3$ uptake, without depleting the complement components, by 70.6-93.4\%. The maximum inhibition of $\mathrm{C3}$ uptake occurred within hours after IVIG infusion, started to rebound $2 \mathrm{~d}$ later, and reached pretreatment levels after $\mathbf{3 0}$ d. The serum levels of SC5b-9 complex production were high at baseline but normalized after IVIG therapy. Repeat biopsies from muscles of improved patients showed disappearance of C3b NEO and MAC deposits from the endomysial capillaries and restoration of the capillary network. We conclude that IVIG exerts its beneficial clinical effect by intercepting the assembly and deposition of MAC on the endomysial capillaries through the formation of complexes between the infused immunoglobulins and $\mathrm{C} 3 \mathrm{~b}$, thereby preventing the incorporation of activated $\mathrm{C3}$ molecules into $\mathrm{C5}$ convertase. These findings provide the first serological and in situ evidence that IVIG modulates complement attack in a human disease. (J. Clin. Invest. 1994. 94:1729-1735.) Key words: complement membrane attack complex - complement C3b • immunomodulation • immunoglobulin therapy - autoimmune diseases
\end{abstract}

Address correspondence to Dr. Milan Basta, National Institutes of Health, Bldg. 10, Rm. 11N238, Bethesda, MD 20892.

Received for publication 12 November 1993 and in revised form 22 July 1994.

1. Abbreviations used in this paper: $\mathrm{C} 3 \mathrm{~b} \mathrm{NEO}$, neoantigen expressed on the surface of activated $\mathrm{C} 3$ component upon incorporation into immune complexes; C5b-C9, complement components five through nine; DM, dermatomyositis; IVIG, high-dose intravenous immunoglobulin; MAC, membrane attack complex; NMD, neuromuscular diseases.

The Journal of Clinical Investigation, Inc.

Volume 94, November 1994, 1729-1735

\section{Introduction}

Dermatomyositis (DM), ${ }^{1}$ polymyositis, and inclusion body myositis are three distinct subgroups of acquired muscle diseases collectively called inflammatory myopathies because endomysial inflammation is a common histological characteristic in all three conditions. DM differs from the other two entities not only clinically because of the characteristic rash that accompanies or often precedes the muscle weakness (1), but also immunopathologically on the basis of a complement-mediated endomysial microangiopathy which is characteristic for the disease (2-5). Membrane attack complex (MAC), consisting of activated complement components $\mathrm{C5b}-\mathrm{C} 9$, is deposited on intramuscular capillaries early in the disease and leads sequentially to destruction of endothelial cells, microinfarcts within the muscle fascicles, muscle ischemia, inflammation, and ultimately perifascicular atrophy $(1,4,5)$. Circulating antibodies to endothelial cells have been described in DM patients, but their cytotoxic effect on endothelial cells has not been demonstrated $(6,7)$.

Because of these immune mechanisms, DM is treated with various immunosuppressive agents. One of the new approaches in the treatment of patients with DM unresponsive to conventional therapies has been with high-dose intravenous immunoglobulin (IVIG). IVIG was found to be very effective in DM patients in one controlled (8) and two uncontrolled $(9,10)$ studies. However, its mechanism of action remains unclear.

In an in vitro model called Forssman shock, IVIG has been effective in preventing the complement-mediated immune damage to the pulmonary microvasculature (11). Anti-Forssman antibodies bind to the Forssman antigen present on the endothelial cells and perivascular tissue and trigger the activation of complement through the classical complement pathway (12). IVIG prevents Forssman shock by modulating the complementdependent immunopathology through diverting activated C3 and C4 fragments from the targets cells (11).

The immunopathological similarities between Forssman shock in guinea pigs and DM in humans (both complementmediated microangiopathies that respond to IVIG) prompted us to examine whether the beneficial effect of IVIG in DM patients is also exerted via the same mechanism as in Forssman shock, namely through interference with the complement system. We demonstrate that the sera of DM patients treated with IVIG exhibit marked inhibition of $\mathrm{C} 3$ uptake resulting in inhibition of the $\mathrm{C} 3 \mathrm{~b}$ deposition on the endomysial capillaries and formation of MAC.

\section{Methods}

Patients and treatment. We studied 13 adult patients with DM who had failed to respond to previous treatments with prednisone, methotrexate, or cyclophosphamide in a double-blind placebo-controlled crossover design. All patients had significant proximal muscle weakness rated 3- 
Table I. Comparison of C3 Uptake Values as Mean Logs before and after IVIG Therapy in Patients with DM and Other NMD

\begin{tabular}{lccccccc}
\hline & \multicolumn{2}{c}{ Pre-IVIG } & & \multicolumn{2}{c}{ Post-IVIG } & Range of post- \\
\cline { 2 - 3 } $\begin{array}{c}\text { Patients } \\
(n=17)\end{array}$ & $\begin{array}{c}\text { Mean } \\
\log *\end{array}$ & SE & & $\begin{array}{c}\text { Mean } \\
\text { log }\end{array}$ & SE & $\begin{array}{c}\text { IVIG } \\
\text { inhibition }\end{array}$ \\
\hline Active DM $(n=9)$ & 4.086 & 0.084 & 3.350 & $0.054^{\ddagger}$ & $70.6-93.4 \%$ \\
Chronic DM $(n=3)$ & 3.298 & 0.039 & & 3.249 & $0.039^{\S}$ & $0-2.3 \%$ \\
Other NMD $(n=5)$ & 3.524 & 0.040 & 3.379 & $0.033^{\prime \prime}$ & $0-4.1 \%$
\end{tabular}

* All values calculated and expressed based on triplicate measurements of $\mathrm{C} 3$ uptake in counts per minute. ${ }^{\ddagger}$ Comparison between pre-IVIG and post-IVIG values; $t=4.31,16 \mathrm{df}, P<0.001$. ${ }^{8}$ Pre-IVIG/postIVIG comparison; $t=0.70,4 \mathrm{df}, P>0.20$. "Pre-IVIG/post-IVIG comparison; $t=2.00,8 \mathrm{df}, P=0.08$.

4/5 MRC scale and a diagnostic muscle biopsy before enrollment. The patients were admitted to the National Institutes of Health Clinical Center after signing an Informed Consent and were randomized to receive three monthly infusions of IVIG at $2 \mathrm{~g} / \mathrm{kg}$ for $2 \mathrm{~d}$ or placebo consisting of dextrose in half-normal saline. Assessment of improvement was based on quantitative measurements of muscle strength and neuromuscular symptoms scores, as described (8).

Patients' sera. A total of 55 coded serum samples from the following patients and controls were examined: patients with DM, before and after treatment with IVIG ( 13 patients) or placebo ( 5 patients); patients with other neuromuscular diseases, such as amyotrophic lateral sclerosis, demyelinating polyneuropathies, or polymyositis before and after IVIG therapy ( 5 patients); and 5 normal controls. The readings in an in vitro C3 uptake assay described later were obtained without prior knowledge of whether a sample was from a treated patient, a control, or whether the patient had received IVIG or placebo.

Antibodies. Monoclonal antibody against C3b NEO human antigen was purchased from Quidel (San Diego, CA). Rabbit anti-human erythrocyte IgG was obtained from Cappel Laboratories (West Chester, PA). Anti-human C3 IgG polyclonal antibody was obtained by immunizing a goat with purified human C3. IgG fractionation was performed by octanoic acid precipitation (13). Anti-MAC monoclonal antibody was a kind gift of Dr. Michaels (University of Minnesota, Minneapolis, $\mathrm{MN}$ ).

Buffers. Isotonic Veronal-buffered saline containing $0.1 \%$ gelatin, $0.15 \mathrm{mmol} /$ liter $\mathrm{Ca}^{2+}$, and $1 \mathrm{mmol} /$ liter $\mathrm{Mg}^{2+}\left(\mathrm{GVBS}^{2+}\right)$, isotonic Veronal-buffered saline containing $0.1 \%$ gelatin, and $10 \mathrm{mmol} / \mathrm{liter}$ EDTA buffer were prepared as described previously (14). Isotonic PBS, pH 7.4, was purchased from Biofluids, Inc. (Rockville, MD).

Other reagents. Sodium $\left({ }^{125} \mathrm{I}\right)$ iodide was purchased from Amersham Corp. (Arlington Heights, IL). Iodobeads were purchased from Pierce Chemical Co. (Rockford, IL).

Radiolabeling. Radioiodination of goat anti-human C3 was accomplished with iodobeads following the directions supplied by the manufacturer. $300 \mu \mathrm{g}$ of the polyclonal IgG was incubated with $1 \mathrm{mCi}$ of iodine-125 and three iodobeads for $30 \mathrm{~min}$ at room temperature. Free iodine was separated from protein-bound isotope by passing the labeled material through a prepacked PD10 (Sephadex G-25M) column (Pharmacia Fine Chemicals, Piscataway, NJ) in isotonic PBS, pH 7.4.

C3 uptake assay. Human red blood cells of type $\mathrm{O}^{+}$were separated from EDTA plasma, washed, and standardized. Aliquots of three-tenths of $1 \mathrm{ml}$ of human red blood suspension at $1 \times 10^{9}$ cells $/ \mathrm{ml}$ were incubated with $0.3 \mathrm{ml}$ of diluted goat IgG anti-human erythrocyte antibody or $0.3 \mathrm{ml}$ of buffer (negative control) for $30 \mathrm{~min}$ at $37^{\circ} \mathrm{C}$. After two washes in $\mathrm{GVBS}^{2+}$, tubes were placed on ice. Pellets of aliquoted sensitized erythrocytes were resuspended in $500 \mu \mathrm{l}$ of undiluted sera from DM patients before and after IVIG therapy or placebo. Pellet of unsensitized erythrocytes was resuspended in normal human serum of type $\mathrm{O}^{+}$. The tubes were then placed in a water bath at $37^{\circ} \mathrm{C}$, and after 5 min of incubation triplicate $60-\mu 1$ samples were removed from each
Table II. Geometric Means, Range, and Mean Logs of Pre-IVIG C3 Uptake Values in Active and Chronic DM, Other NMD, and Normal Controls

\begin{tabular}{lccc}
\hline \multicolumn{1}{c}{$\begin{array}{c}\text { Patients } \\
(n=22)\end{array}$} & $\begin{array}{c}\text { Geometric } \\
\text { means* }\end{array}$ & \multicolumn{1}{c}{ Range } & $\begin{array}{c}\text { Mean } \\
\text { log }\end{array}$ \\
\hline Active DM $(n=9)$ & 12,190 & $2,032-61,646$ & $4.086^{\ddagger}$ \\
Chronic DM $(n=3)$ & 1,986 & $1,418-2,220$ & 3.298 \\
Other NMD $(n=5)$ & 3,342 & $1,993-5,442$ & 3.524 \\
Normals $(n=5)$ & 3,724 & $1,549-10,914$ & 3.571 \\
\hline
\end{tabular}

* All values calculated and expressed based on triplicate measurements of C3 uptake in counts per minute. ${ }^{\ddagger}$ Comparison between active DM and other conditions; $t=2.28,18 \mathrm{df}, P<0.05$.

tube and transferred to corresponding tubes with $2 \mathrm{ml}$ of ice-cold EDTA buffer. The erythrocytes in these samples were pelleted and washed once in $\mathrm{GVBS}^{2+}$. The final pellets were resuspended in $200 \mu \mathrm{l}$ of $\mathrm{GVBS}^{2+}$. Each tube received the amount of radiolabeled anti-C3 antibody equal to $2 \times 10^{6} \mathrm{cpm}$ and incubated on melting ice for $60 \mathrm{~min}$ with occasional shaking. After two further washes in ice-cold EDTA buffer, the pellets were counted in a gamma counter.

$S C 5 b-9$ ELISA assay. The amount of the SC5b-9 complexes present in serum samples was measured by ELISA using a commercially available kit (Quidel) following the manufacturer's instructions.

Muscle immunohistochemistry. Two muscle biopsies, one from each biceps muscle, were performed in five patients, one before starting IVIG therapy and the other upon completion of three monthly infusions. The patients were selected for repeat biopsies based on a convincing objective clinical response to IVIG therapy in terms of normalization of muscle strength (from 3-4/5 MRC scale before therapy to $5 / 5$ after treatment) before the code was broken ( 8 ). The muscle biopsy specimens were processed simultaneously for immunocytochemistry as described $(8,15)$. Briefly, three $4-\mu \mathrm{m}$ serial sections of fresh-frozen muscle biopsies were fixed in acetone for $5 \mathrm{~min}$ at $4^{\circ} \mathrm{C}$, rinsed in $0.05 \mathrm{M}$ Tris-buffered saline ( $\mathrm{pH} \mathrm{7.6)}$ ) for $15 \mathrm{~min}$, and incubated for $30 \mathrm{~min}$ with a blocking solution containing $1 \%$ bovine serum albumin, $10 \%$ normal human serum, and 3\% normal horse serum. The sections were incubated with the primary mouse monoclonal antibody anti-MAC at 1:40 dilution or anti-C3b NEO at 1:60 dilution. To determine if the MAC was on the muscle fibers or the capillaries, the same sections were subjected to dual immunostaining with anti-MAC followed by FITC anti-mouse IgG, and with a capillary marker, the biotinylated lectin Ulex Europeus (Vector Labs, Inc., Burlingame, CA) followed by avidin-rhodamine, in single and double exposure settings, as described $(1,3)$. The C $3 \mathrm{~b}$ NEO deposition was identified by single staining procedure using biotinylated secondary antibody and avidin-biotin-peroxidase complex (Vector Labs, Inc.). Antigen-antibody reaction was visualized by incubating the tissue sections with $0.05 \%$ (wt/vol) 3,3-diaminobenzidine.

Statistical analysis. Analysis of variance was performed by use of PROC GLM procedure of the computer software SAS/STAT (SAS Institute, Cary, NC). Log normal distribution was tested by ShapiroWilk test (16).

\section{Results}

In vitro C3 uptake by sera of patients with DM and controls. To determine the effect of IVIG therapy on the ability of the patients' sera to deposit activated C3 fragments onto sensitized human erythrocytes, serum samples (collected from each patient before administration of IVIG therapy and $2 \mathrm{~d}$ after completion of the first dose) were screened in the in vitro C3 uptake assays. After IVIG therapy, C3 uptake (expressed as log mean of triplicate measurements of counts per minute) was decreased to a highly significant degree in patients with active DM com- 


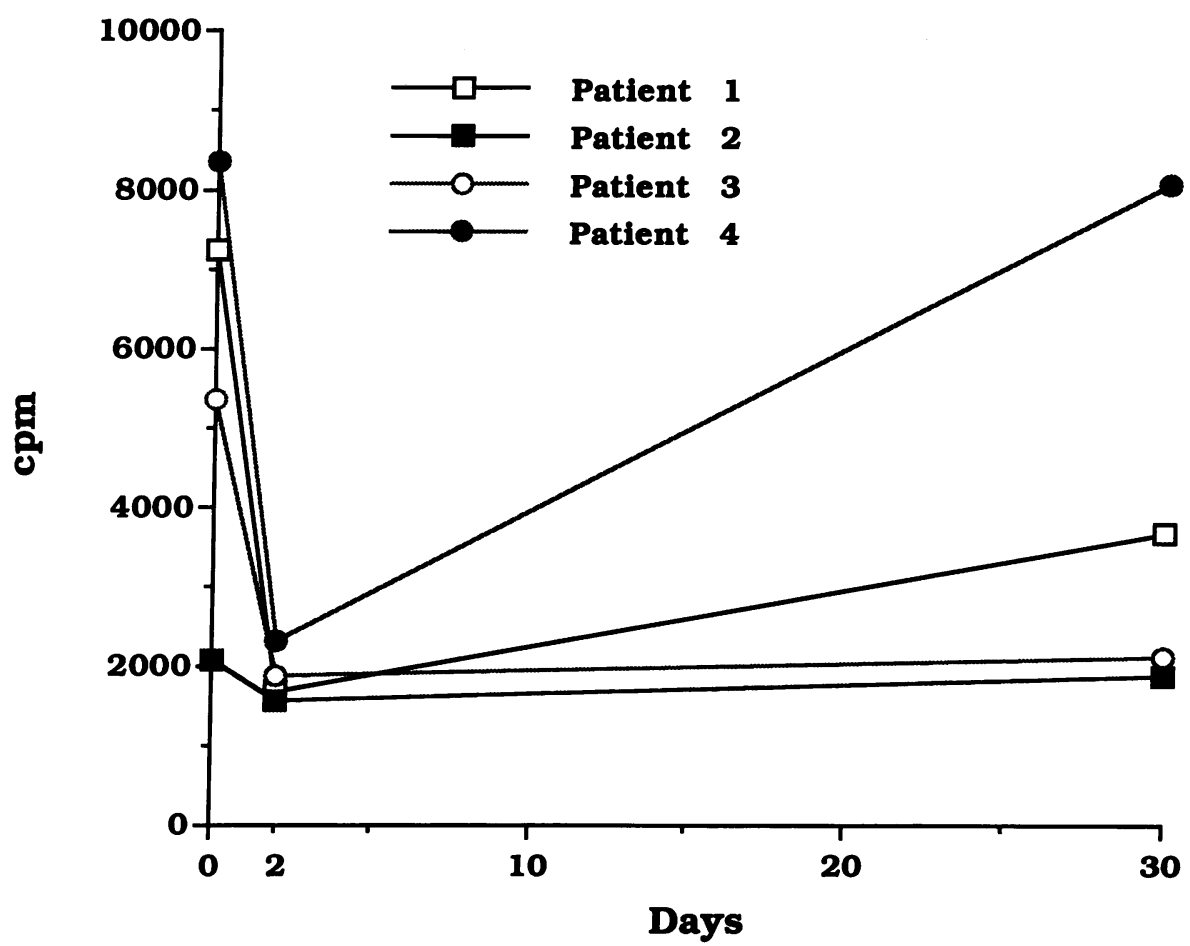

B

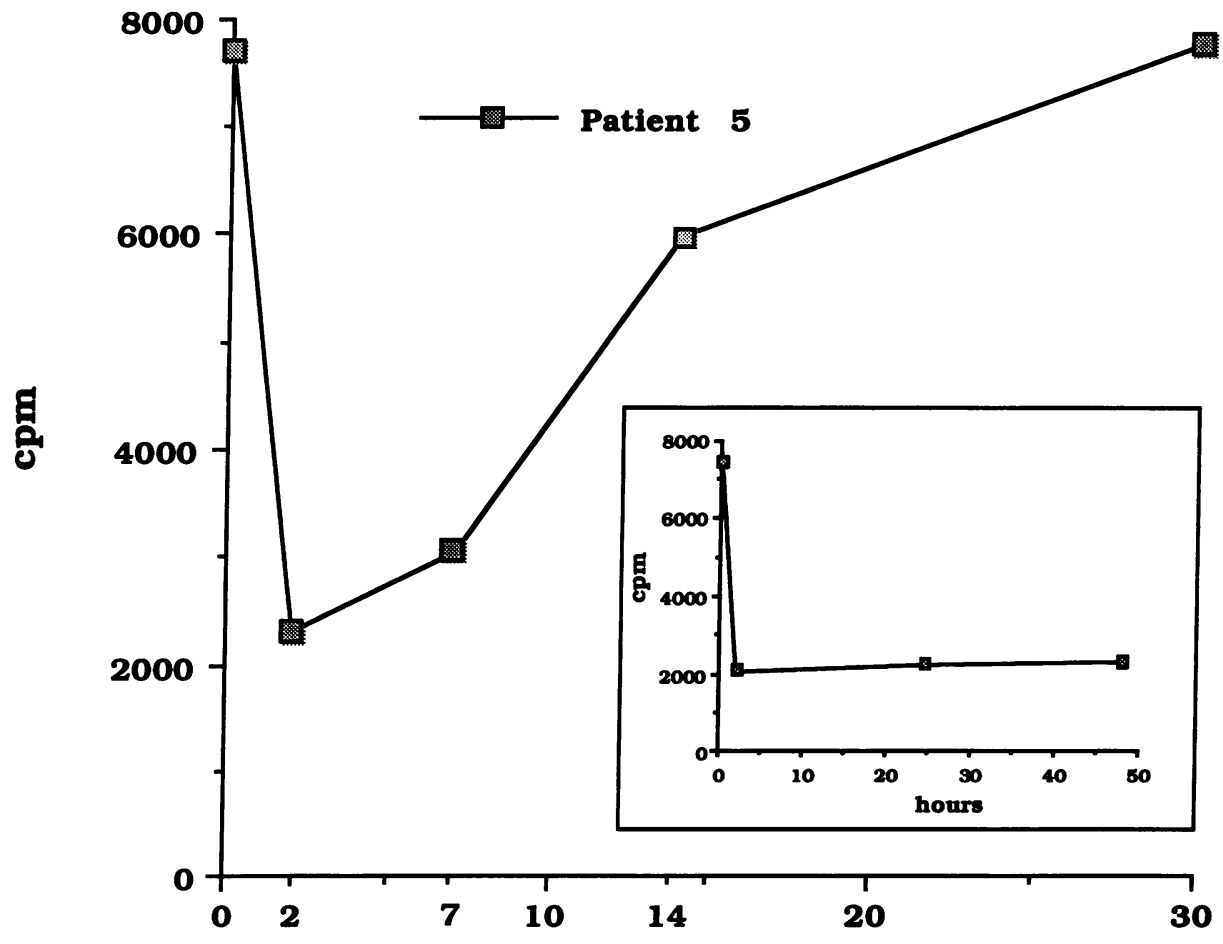

Days

Figure 1. Kinetics of $\mathrm{C} 3$ uptake in sera of DM patients after IVIG therapy. C3 uptake was quantitated by use of ${ }^{125} \mathrm{I}$-labeled antihuman $\mathrm{C} 3$ antibody in serum samples of four patients at baseline, 2 d after completion of IVIG therapy, and $30 \mathrm{~d}$ later. At day 2 after IVIG infusion, the C3 uptake in all the patients was suppressed to the background level; $30 \mathrm{~d}$ later, the C3 uptake had rebounded and risen to $97 \%$ of the baseline in patient 4 , up to $90 \%$ in patient 2 , up to $40 \%$ in patient 3 , and $51 \%$ in patient 1 . The $\mathrm{C} 3$ uptake was measured serially in another patient (patient 5), at baseline and at 2, 24 , and $48 \mathrm{~h}$ after IVIG infusion $(B$, inset $)$, as well as at 7,14 , and $30 \mathrm{~d}$ post-IVIG $(B)$. C3 uptake was inhibited to background $2 \mathrm{~h}$ after IVIG infusion and remained unchanged until day $2(B$, inset $)$. Eventually, C3 uptake reached the pre-IVIG (baseline) value at day $30(B)$.

pared with the same patients' sera before IVIG therapy ( $t$ $=4.31,16 \mathrm{df}, P<0.001$ ) (Table I). The percentage of inhibition varied in individual patients from 70.6 to $93.4 \%$ and was more pronounced in the patients with the highest baseline (pre-
IVIG) C3 uptake (not shown). The patients who had the highest percentage of inhibition also had major clinical improvement, as judged by the increase of muscle strength and disappearance of rash (8). Comparisons of pre-IVIG and post-IVIG C3 uptake 

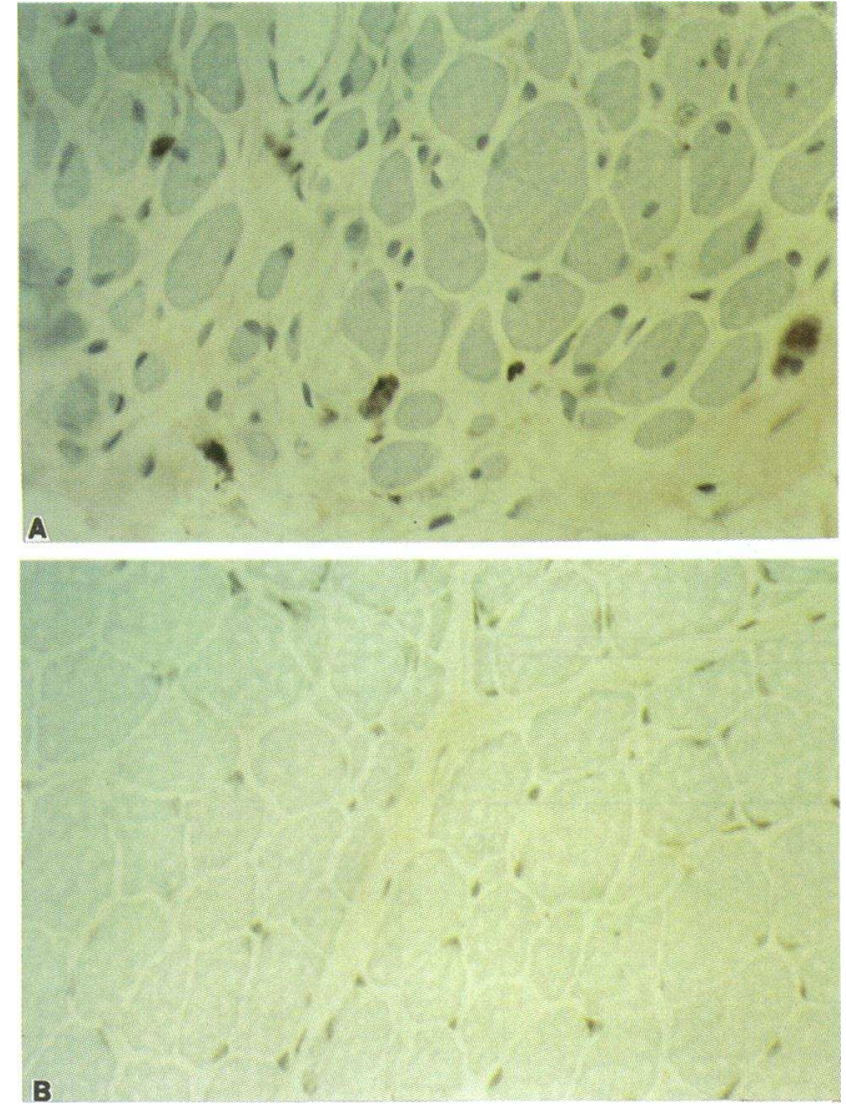

Figure 2. Immunostaining of muscle biopsy specimens from DM patients for C3b NEO antigen. 4- $\mu$ m-thick muscle biopsy sections from a patient with active DM before IVIG infusions $(A)$ and upon completion of three monthly infusions of IVIG $(B)$ immunostained with antibody against $C 3 b$ NEO followed by biotinylated secondary antibody and avidin-biotin-peroxidase complex. In the pre-IVIG biopsy, C3b NEO is deposited on the muscle capillaries and some muscle fibers $(A)$; in the post-IVIG specimen, no C3b NEO deposits were detected $(B) . \times 375$.
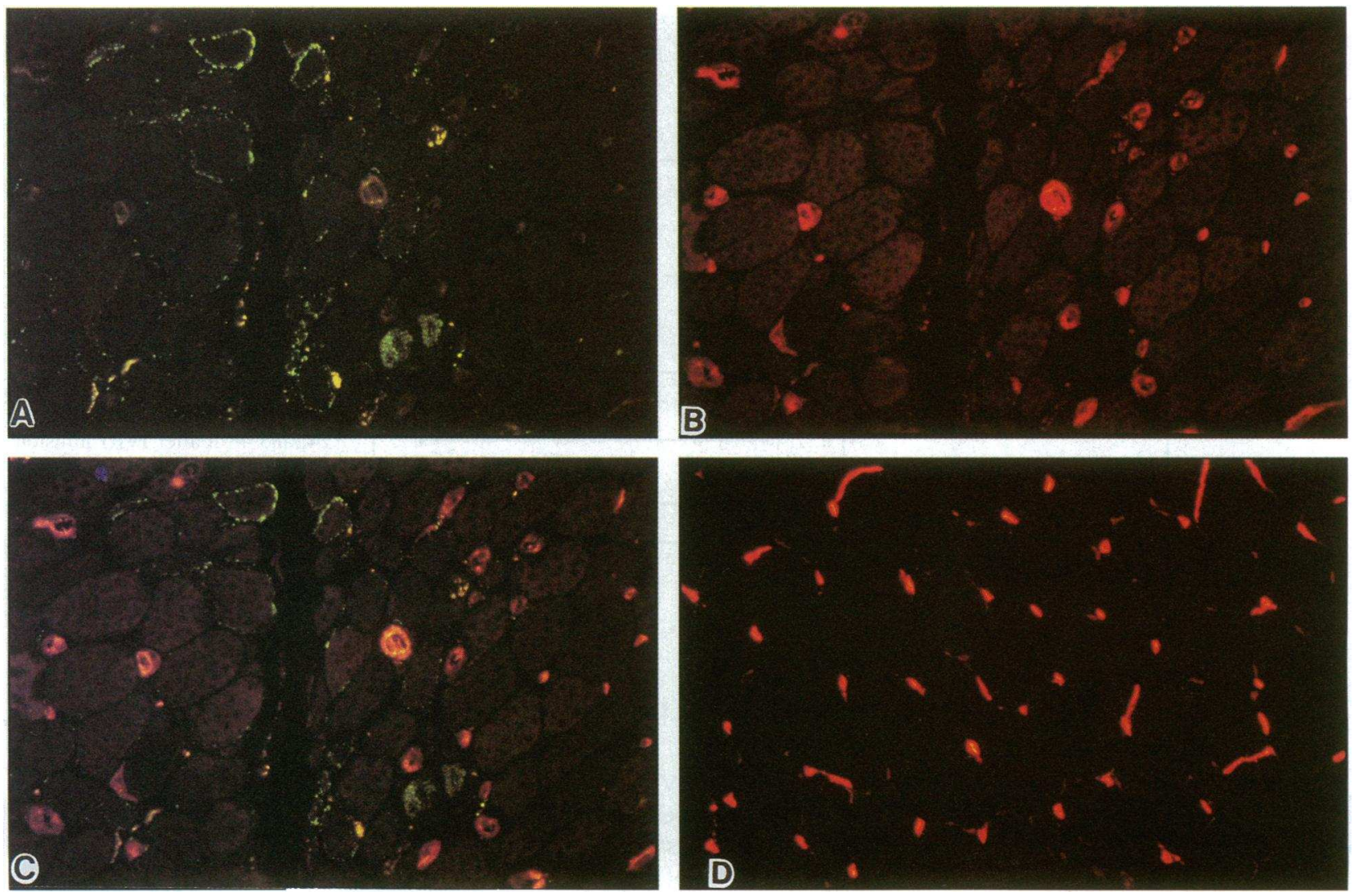
in patients with chronic, long-lasting DM ( $t=0.70,4 \mathrm{df}, P>$ $0.20)$ and patients with other neurological diseases $(t=2.00$, $8 \mathrm{df}, P=0.08$ ) did not reveal any significant difference (Table I). No statistically significant difference was found in coded samples from DM patients before and after placebo, run simultaneously $(t=0.18,8 \mathrm{df}, P>0.20)$.

Analysis of variance between baseline C3 uptake in patients with active progressive disease and pooled baseline data from patients with chronic DM, other neurological diseases, and normals showed significantly higher uptake in the active DM ( $t$ $=2.28,18 \mathrm{df}, P<0.05)$. Pooling of groups for statistical analysis was justifiable because of the intergroup homogeneity, i.e., no significant difference between their mean logs (Table II). Pooling was necessary because of the small numbers in control groups. All patient and control groups fit the log normal distribution as determined by Shapiro-Wilk test (16) (not shown).

Detection of $S C 5 b-9$ in patients sera. The presence of the SC5b-9 complex in sera of nine DM patients and five normal controls was studied quantitatively using a commercially available ELISA assay (Quidel). High levels of SC5b-9 were detected in the serum of DM patients before IVIG therapy, ranging from 250 to $640 \mathrm{ng} / \mathrm{ml}$, compared with $115-190 \mathrm{ng} / \mathrm{ml}$ of the normal controls. In post-IVIG samples, the amount of SC5b-9 was suppressed to nearly normal level, with values ranging from 140 to $225 \mathrm{ng} / \mathrm{ml}$.

C3 uptake in serum samples before IVIG and at various times after therapy. C3 uptake was measured in the serum of four patients with active DM sequentially, before IVIG infusion, $2 \mathrm{~d}$ after completion of IVIG therapy, and $30 \mathrm{~d}$ later (before the next monthly IVIG infusion). C3 uptake was inhibited to almost background values $2 \mathrm{~d}$ after IVIG (Fig. $1 A$ ). $30 \mathrm{~d}$ later the $\mathrm{C} 3$ uptake returned to baseline in one patient and rebounded by $40-90 \%$ in the other three (Fig. $1 \mathrm{~A}$ ).

In a subsequent patient, the kinetics of C3 uptake inhibition were examined closely, using more time points. The C3 uptake was inhibited to the background level $2 \mathrm{~h}$ after IVIG infusion and remained at that level for $2 \mathrm{~d}$ (Fig. $1 \mathrm{~B}$, inset). After the second day post-IVIG, the C3 uptake started to increase slowly, reaching baseline value by day 30 (Fig. $1 \mathrm{~B}$ ).

Immunohistochemical staining for $C 3 b$ NEO antigen in biopsy specimens before and after IVIG therapy. To determine if the effect on C3 uptake can be correlated with in situ events related to $\mathrm{C} 3$ deposition into immune complexes on capillaries at sites of muscle damage, we immunostained muscle biopsy specimens with the monoclonal antibody against a new epitope (neoantigen) of $\mathrm{C} 3 \mathrm{~b}$ that gets exposed upon the incorporation of $\mathrm{C} 3 \mathrm{~b}$ into an immune complex; C3b NEO antigen is therefore immune complex-specific for this activated C3 fragment (17). Before IVIG infusion, the C3b NEO antigen was detected on up to $10 \%$ of the muscle capillaries and on occasional (1-5) muscle fibers per field (at a magnification of 25) (Fig. $2 A$ ). After IVIG therapy, no C3b deposits were detected (Fig. $2 B$ ). Furthermore, there was a significant histological improvement after IVIG with increase in the size of the muscle fibers, especially at the periphery of the fascicle (Fig. $2 B$ ), as described previously (8).

The effect of IVIG therapy on the deposition of MAC of the complement cascade in muscle capillaries. The formation of MAC is the function of C5 convertase formation for which incorporation of $\mathrm{C} 3 \mathrm{~b}$ is essential; any change in $\mathrm{C} 3 \mathrm{~b}$ uptake affects the MAC formation exponentially (18). For this reason, we examined the deposition of MAC in muscle fiber capillaries before and after IVIG therapy. Staining for MAC (Fig. $3 A$ ) and endothelial cells (Fig. $3 B$ ) before the institution of IVIG therapy showed by dual exposure (Fig. $3 C$ ) that MAC complexes were on the endothelial cells and muscle fibers. After high dose IVIG, no MAC deposits were detected (Fig. $3 \mathrm{D}$ ).

Degree of clinical improvement in patients with high $C 3$ uptake inhibition. As reported previously (8), when the total MRC and the total neuromuscular symptom score increased by five or more grades each, the patients had a major improvement. All the patients with active DM, whose sera showed the highest C3 uptake inhibition, had major clinical improvement. Their mean MRC score increased from 74.5 \pm 5.9 at baseline to $84.7 \pm 4.5(P<0.018)$, and the mean neuromuscular symptom score increased from $38.6 \pm 5.9$ to $51.0 \pm 8.0(P<0.035)(8)$. In contrast, the $\mathrm{C} 3$ uptake inhibition and the neuromuscular symptom score remained unchanged in the patients who received placebo.

\section{Discussion}

IVIG has been found to be effective in a variety of autoimmune cytopenias (19-21) and other immune-mediated disorders (22, 23). The exact mechanisms of action of IVIG, however, have not been elucidated. The proposed theories include: $(a)$ blockade of IgG Fc receptors on phagocytic cells by the infused IVIG, thereby preventing the removal from the circulation of cells sensitized with autoantibodies of IgG isotype (24); (b) presence of antiidiotypic antibodies capable of suppressing autoantibody secretion in the pool of plasma donors used for IVIG production (25); $(c)$ direct effect on $\mathrm{B}$ and $\mathrm{T}$ lymphocytes $(26) ;(d)$ inhibition of cytokine production $(27,28) ;(e)$ the presence of soluble CD4, CD8, and HLA that modify antigen recognition by target cells (29); and $(f)$ interference with the complement system. The last theory was based on two in vivo models of complement-mediated immune damage $(8,30)$ and several in vitro complement-uptake assays $(31,32)$. These studies have shown that IVIG can effectively modulate the com-

Figure 3. Transverse section of a fresh-frozen muscle biopsy specimen from a patient with DM before and after IVIG. Cross-sections of freshfrozen muscle biopsy specimens from a patient with DM dually immunostained with antibodies to the C5b-9 MAC and fluorescein isothiocyanateconjugated anti-mouse IgG, which highlights the complement deposits with a green fluorescence $(A)$, and with biotinylated Ulex Europeus and avidin-rhodamine, which highlights the endomysial capillaries with a red fluorescence $(B)$. The double exposure of $A$ and $B$ produces an orange color $(C)$ from superimposition of the green fluorescence from the MAC deposits and the red fluorescence on the capillary wall. Before IVIG therapy, MAC deposits are present not only on the capillaries (orange color in $C$ ) but also on some perifascicular muscle fibers (green color in $A$ and $C$ ). After IVIG therapy, the repeat specimen dually immunostained for MAC and Ulex $(D)$ shows absence of MAC deposits (no green fluorescence visualized) from both the capillaries and the muscle fibers, in a randomly selected perifascicular region (periphery of the fascicle is at the lower right). Note the increase in the number of capillaries $(D)$ and the decrease in their diameter compared with the pretreatment specimen $(B) . \times 250$. 
plement-dependent immunopathology by diverting activated complement fragments, $\mathrm{C} 3 \mathrm{~b}$ and $\mathrm{C} 4 \mathrm{~b}$, from target cells, thus preventing the complement-mediated destruction of microvasculature. This action is relevant to the beneficial effect of IVIG in patients with DM where an MAC-mediated destruction of the endomysial capillaries is the major pathogenic event $(4,5)$.

Our study presents evidence that the sera of DM patients after IVIG therapy markedly suppressed the uptake of C3 fragments onto targets in vitro. Furthermore, IVIG almost abrogated the deposition of $\mathrm{C} 3 \mathrm{~b}$ in immune complexes and the MAC deposits on the target cells within the muscle after the full course of IVIG therapy. These findings collectively indicate that the remarkable clinical and histological improvement observed in DM patients is due to the interception of MAC formation at the level of C5 convertase assembly, caused by interaction between the Ig molecules of IVIG and the C3b fragments. The mechanism of this interaction is most likely the formation of covalent or noncovalent complexes between specific acceptor site(s) within the immunoglobulin molecule and C3b. This interception results in unavailability of $\mathrm{C} 3 \mathrm{~b}$ for incorporation into the enzyme C5 convertase which is necessary for triggering MAC, as supported by the absence of MAC from the endomysial capillaries in the repeat biopsies. The absence of MAC deposits on the capillaries allowed neovascularization, reperfusion of the muscle, resolution of muscle ischemia, and finally increased muscle strength, as reported (8).

The beneficial effect of IVIG via the complement pathway is supported by the reversal of the inhibition of C3 uptake in the sera of DM patients $30 \mathrm{~d}$ after IVIG therapy, consistent with the half-life of the infused IgG, and by the lack of effect of the placebo-treated sera in the C3 uptake. Therapy itself did not cause a decrease in function or availability of complement components, since $\mathrm{C} 3$ antigen and $\mathrm{CH} 50$ values remained within the normal range in both pre- and post-IVIG samples. By using coded sera, the interpretation and documentation of C3 uptake in the sera subjected to analysis were unbiased. Although the effect of IVIG on C4 uptake was not studied, our previous studies have shown that IVIG inhibits the C4 uptake to the same degree as C3 (31).

The finding of high baseline (pre-IVIG) C3 uptake values in patients with the most active form of DM supports the complement-mediated mechanism of the disease. This is further strengthened by correlation of disease activity with the activation of complement and generation of active fragments of early complement components ( C3b, C4b). The increased C3 uptake in active phase of DM, but not in the chronic form or the controls, suggests that the high rate of complement activation may be responsible for the formation of a large number of activated early components. Whether C3 uptake is also increased in other complement-mediated neuromuscular diseases, such as Guillain-Barré syndrome (33) or myasthenia gravis where IVIG is also effective $(23,34)$, is not known.

Our results may provide an explanation for the mechanism of therapeutic effect of IVIG in other conditions in which complement-mediated tissue injury plays a role in their cause.

\section{Acknowledgments}

The authors thank Dr. David Alling for performing the statistical analysis of data, Dr. Monzon for her help with radiolabeling of anti-human C3 antibody, and Dr. Illa for help with the double immunocytochemistry.

\section{References}

1. Dalakas, M. C. 1991. Polymyositis, dermatomyositis, and inclusion-body myositis. N. Engl. J. Med. 325:1487-1498.

2. Dalakas, M. C. 1992. Inflammatory and toxic myopathies. Curr. Opin. Neurol. Neurosurg. 5:645-654.

3. Dalakas, M. C. 1992. Clinical, immunopathological, and therapeutic considerations of inflammatory myopathies. Clin. Neuropharmacol. 15:327-351.

4. Kissel, J. T., J. R. Mendell, and K. W. Rammohan. 1986. Microvascular deposition of complement membrane attack complex in dermatomyositis. N. Engl. J. Med. 314:329-334.

5. Emslie-Smith, A. M., and A. G. Engel. 1990. Microvascular changes in early and advanced dermatomyositis: a quantitative study. Ann. Neurol. 27:343356.

6. Cervera, R., G. Ramirez, J. Fernandez-Sola, D. D'Cruz, J. Casademont, J. M. Grau, R. A. Asherson, M. A. Khamashta, A. Urbano-Marquez, and G. R. V. Hughes. 1991. Antibodies to endothelial cells in dermatomyositis: association with interstitial lung disease. Br. Med. J. 302:880-881.

7. Stein, D. P., S. C. Jordan, M. Toyoda, O. Gallera, and M. C. Dalakas. 1993. Anti-endothelial cell antibodies (AECA) in dermatomyositis (DM). Neurology. 43 (Suppl):356a. (Abstr.)

8. Dalakas, M. C., I. Illa, J. M. D'Ambrosia, S. A. Soueidan, D. P. Stein, C. Otero, S. T. Dinsmore, and S. McCrosky. 1993. A controled trial of high-dose immune globulin infusions as treatment for dermatomyositis. N. Engl. J. Med. 329:1993-2000.

9. Cherin, P., S. Herson, B. Wechsler, J. C. Piettre, O. Bletry, J. M. Ziza, C. Degennes, and P. Godeau. 1991. Effect of intravenous gammaglobulin therapy in chronic refractory polymyositis and dermatomyositis. An open study with 20 adult patients. Am. J. Med. 91:162-168.

10. Lang, B. A., R. M. Laxer, G. Murphey, E. D. Silverman, and C. M. Roifman. 1991. Treatment of dermatomyositis with intravenous gammaglobulin. Am. J. Med. 91:169-172.

11. Basta, M., P. Kirshbom, M. M. Frank, and L. F. Fries. 1989. Mechanism of therapeutic effect of high-dose intravenous immunoglobulin. Attenuation of acute, complement-dependent immune damage in a guinea pig model. J. Clin. Invest. 84:1974-1981.

12. May, J. E., and M. M. Frank. 1972. Complement-mediated tissue damage: contribution of the classical and alternative complement pathways in the Forssman reaction. J. Immunol. 108:1517-1525.

13. Steinbuch, M., and R. Audran. 1969. The isolation of IgG from mammalian sera with the aid of caprylic acid. Arch. Biochem. Biophys. 134:279-284.

14. Gaither, T. A., and M. M. Frank. 1979. Complement. In Clinical Diagnosis and Management by Laboratory Methods. J. B. Henry, editor. W. B. Saunders Co., Philadelphia. 1253.

15. Illa, I., M. E. L. Leon-Monzon, and M. C. Dalakas. 1992. Regenerating and denervated human muscle fibers and satellite cells express neural cell adhesion molecule recognized by monoclonal antibodies to natural killer cells. Ann. Neurol. 31:46-52.

16. Shapiro, S. S., and M. B. Wilk. 1965. An analysis of variance test for normality (complete and samples). Biometrika. 52:591-611.

17. Aguado, M. T., J. D. Lambris, G. C. Tsokos, R. Burger, D. Bitter-Suermann, J. D. Tamerius, F. J. Dixon, and A. N. Theofilopoulos. 1985. Monoclonal antibodies against complement 3 neoantigens for detection of immune complexes and complement activation. J. Clin. Invest. 76:1418-1426.

18. Frank, M. M., and L. F. Fries. 1989. Complement in Fundamental Immunology. 2nd ed. W. E. Paul, editor. Raven Press, New York. 688-690.

19. Imbach, P., S. Barandun, V. d'Apuzzo, A. Hirt, E. Rossi, M. Vest, C. Baumgarten, A. Morel, and H. P. Wagner. 1981. High-dose intravenous gammaglobulin for idiopathic thrombocytopenic purpura in childhood. Lancet. i:12281230 .

20. Pocecco, M., A. Ventura, P. Tamaro, and F. Longo. 1985. High-dose IVIgG in autoimmune hemolytic anemia (Evans syndrome). J. Pediatr. 107:744746.

21. Goldstein, R., V. S. Blanchette, L. B. Huebsch, and R. J. R. McKendry. 1986. Treatment of gold-induced thrombocytopenia by high-dose intravenous immunoglobulin. Arthritis Rheum. 29:426-430.

22. Newberg, N. J., M. Takahashi, and J. Burns. 1986. The treatment of Kawasaki syndrome with intravenous gamma globulin. N. Engl. J. Med. 315:341 347.

23. Arsura, E. L., A. Bick, N. G. Bruner, T. Namba, and D. Grob. 1986. Highdose intravenous immunoglobulin in the management of myasthenia gravis. Arch. Intern. Med. 146:1365-1368.

24. Kurlander, R. J., D. M. Ellison, and J. Hall. 1984. The blockade of Fc receptor-mediated clearance of immune complexes in-vivo by monoclonal antibody (2.4G2) directed against Fc receptors on murine leukocytes. J. Immunol. 133:855-862.

25. Rossi, F., Y. Sultan, and M. D. Kazatchkine. 1986. Spontaneous and therapeutic suppression of autoimmune response to factor VIII by anti-idiotypic antibodies. In Clinical Use of Intravenous Immunoglobulins. A. Morell and U. E. Nydegger, editors. Academic Press Ltd., London. 421.

26. Durandy, A., A. Fisher, and C. Griscelli. 1981. Dysfunctions of pokeweed 
mitogen-stimulated $\mathrm{T}$ and $\mathrm{B}$ lymphocyte responses induced by gammaglobulin therapy. J. Clin. Invest. 67:867-877.

27. Andersson, J. P., and U. G. Andersson. 1990. Human intravenous immunoglobulin modulates monokine production in vitro. Immunology. 71:372-376.

28. Leung, D. Y. M., E. Kurt-Jones, J. W. Newberger, R. S. Cotran, J. C. Burns, and J. S. Pober. 1989. Endothelial cell activation and high interleukin-1 secretion in the pathogenesis of acute Kawasaki disease. Lancet. ii:1298-1302.

29. Blasczyk, R., U. Westhoff, and H. Grosse-Wilde. 1993. Soluble CD4, CD8, and HLA molecules in commercial immunoglobulin preparations. Lancet. 341:789-790.

30. Basta, M., P. F. Langlois, M. Marques, M. M. Frank, and L. F. Fries. 1989. High-dose intravenous immunoglobulin modifies complement-mediated invivo clearance. Blood. 74:326-333.
31. Basta, M., L. F. Fries, and M. M. Frank. 1991. High-dose intravenous immunoglobulin (IVIG) inhibit in vitro uptake of C4 fragments onto sensitized erythrocytes. Blood. 77:376-380.

32. Basta, M., L. F. Fries, and M. M. Frank. 1991. High doses of intravenous immunoglobulin do not affect the recognition phase of the classical complement pathway. Blood. 78:700-702.

33. Koski, C. L., M. E. Sanders, P. T. Swoveland, T. J. Lawley, M. L. Shin, M. M. Frank, and K. A. Joiner. 1987. Activation of terminal components of complement in patients with Guillain-Barré syndrome and other demyelinating neuropathies. J. Clin. Invest. 80:1492-1497.

34. Van Der Meche, F. G., P. I. Schmitz, and Dutch Guillain-Barre Study Group. 1992. A randomized trial comparing intravenous immune globulin and plasma exchange in Guillain-Barré syndrome. N. Engl. J. Med. 39:422-425. 